\title{
Kriegerische Maskulinitätskonstrukte und sexualisierte Gewalt in Sierra Leone und Uganda
}

\author{
Rita Schäfer*
}

\begin{abstract}
For analyses of gender and security as well as for political planning regarding improvements of specific security situations in post-war societies, it is important to understand gender-specific violence as a structural problem. Differences between men and women should be considered thereby. Only then does it become clear, who among the men are often the actors of violence and who among the women are primarily threatened by acts of violence.
\end{abstract}

Keywords: Kriegsgeprägte Maskulinität, sexualisierte Gewalt, Nachkriegsgesellschaften

Militant masculinity, sexual violence, post-conflict societies

\section{Einleitung}

In der Kontroverse über das Konzept „menschliche Sicherheit“ sowie dessen politische Umsetzung verlangen Forscherinnen seit Jahren eine differenzierte Auseinandersetzung mit den Geschlechterdimensionen. Trotz gegensätzlicher Positionen zur Verknüpfung von Sicherheitsinteressen mit sozio-ökonomischen und politischen Strukturproblemen sowie zur Frage der (Un-)Vereinbarkeit grundlegender Menschenrechte mit dem neuen Sicherheitskonzept prangern Befürworterinnen wie Gegnerinnen einhellig die hohen Raten an häuslicher und sexueller Gewalt in Nachkriegsgesellschaften an. Unter Bezug auf klar formulierte oder subtil verborgene feministische Positionen gehen sie davon aus, dass alle Frauen in ähnlicher Weise von Gewalt bedroht sind. Dabei ignorieren sie vielfältige Differenzen zwischen Frauen, z.B. auf der Basis des Alters, des familiären Status, der sozio-ökonomischen Position, der religiösen Orientierung, der Herkunft, der ethnischen Zugehörigkeit und der nationalen Identität. Diese Differenzkategorien sind häufig in komplexer Weise miteinander verwoben und verstärken lokale Geschlechterhierarchien; sie spiegeln sich in unterschiedlichen Kriegs- und Nachkriegserfahrungen von Frauen und Mädchen wider und beeinflussen deren konkrete Bedrohung durch geschlechtsspezifische Gewalt.

Immerhin weisen einige Friedensexpertinnen in neueren Studien darauf hin, dass nicht nur Männer aus dem jeweiligen sozialen Umfeld, sondern auch Soldaten der staatlichen Armeen, Polizisten oder Mitglieder von Friedensmissionen zu den Tätern zählen (Ulbert 2008:59f.). Indem diese Arbeiten unterschiedliche männliche Akteursgruppen in den Blick nehmen, brechen sie zumindest ansatzweise verbreitete Geschlechterstereotypen und Vorstellungen von einer homogenen Tätergruppe auf. Aktuelle Forschungen ordnen die Gewaltakte in größere gesellschaftliche und politische Kontexte ein. Sie betonen, dass sexuelle Übergriffe durch Polizisten und Soldaten der Legitimität staatlicher Institutionen in Nachkriegsgesellschaften schaden und dem Wiederaufbau von Rechtsstaatlichkeit diametral entgegenstehen. Einzelne junge Forscher decken

Dr. Rita Schäfer ist Ethnologin und Lehrbeauftragte am Institut für Friedenssicherungsrecht und Humanitäres Völkerrecht, Ruhr-Universität Bochum. die Konsequenzen des Gewalthandelns von Staatsdienern und Blauhelmsoldaten für die lokalen Geschlechterhierarchien auf. An empirischen Fallbeispielen weisen sie nach, dass Männer aus der lokalen Bevölkerung sich durch das besitzergreifende Sexualverhalten ausländischer Friedenskräfte in ihrer eigenen Maskulinität angegriffen fühlen (Higate/Henry 2004:483ff.).

Im Folgenden soll die Relevanz der Hierarchien zwischen Männern unterschiedlicher Herkunft als wichtiger Faktor für die hohen Gewaltraten in Nachkriegsgesellschaften an zwei Länderbeispielen - Sierra Leone und Uganda - spezifiziert und um eine neue Betrachtungsebene erweitert werden, nämlich um die Unterschiede zwischen Frauen und zwischen Männern innerhalb einer Gesellschaft. In diesen Fallstudien wird zweierlei deutlich: Erstens sind keineswegs alle Frauen in ähnlicher Weise Gewaltopfer, und zweitens beeinflussen die innergesellschaftlichen Hierarchien zwischen Männern die Gewaltbereitschaft der jeweiligen Täter. In beiden Ländern sind insbesondere junge, sozial marginalisierte Ex-Kombattantinnen mit Gewalt konfrontiert. Im innerfamiliären Kontext zählen vor allem diejenigen Frauen zu Gewaltopfern, deren Ehemänner oder Väter bei Vergewaltigungen durch Gegner zuschauen und die jahrelang in Flüchtlingslagern ausharren mussten. Eine weitere Gruppe von Gewaltakteuren sind junge Ex-Kombattanten, deren kriegerische Gewaltsozialisation und gewaltbereite Männlichkeit in Demobilisierungs- und Reintegrationsprogrammen nicht reduziert werden konnte. Stattdessen nutzen viele das antrainierte Gewalthandeln im Nachkriegsalltag weiterhin zur Interessendurchsetzung, zumal sie durch die Entwaffnungsprogramme in ihrem Selbstbild verunsichert sind. Auch junge Männer, die jahrelang in Flüchtlingscamps leben und von denen etliche zuvor Kämpfer waren, fühlen sich durch bewaffnete Soldaten, die die Lager kontrollieren, entmännlicht. Viele versuchen gewaltsam, ihre Macht nicht nur gegenüber Frauen und Mädchen, sondern auch gegenüber anderen Männern zu beweisen. Um diese Problemkomplexität zu verstehen, sind differenzierte Auseinandersetzungen mit kriegsgeprägten Gender-Konstrukten und Kontextanalysen der gewaltsam aufgeladenen Maskulinität notwendig.

Erkenntnisleitend für die folgende Analyse ist ein umfassender Gender-Begriff, der von gesellschaftlich konstruierten Normen ausgeht und diese zeitlich kontextualisiert. Zudem wird Gender hier als Machtphänomen behandelt, das mit politischen und 
ökonomischen Machtstrukturen interdependent verwoben ist. Diese Machtverhältnisse werden durch verschiedene Institutionen, gesellschaftliche Gruppen und das Handeln Einzelner geprägt, somit werden Gender-Normen in sozialen Interaktionen ausgehandelt. Welche Gefahren diese Dynamik in sich birgt, zeigt die Militarisierung von Männlichkeit vor und während Kriegen. Geschickt manipulieren Politiker, ranghohe Militärs, Kriegsherren und religiöse Autoritäten lokale Männlichkeitskonzepte, indem sie diese mit politisch und kulturell aufgeladenen Bedrohungsszenarien potenzieren; gleichzeitig reduzieren sie maskuline Rollenzuschreibungen auf die Verteidigerrolle. Dabei nutzen die Kriegstreiber grundlegende Probleme aus, etwa die vielfältigen Formen struktureller Gewalt, große soziale Ungleichheiten, hohe Arbeitslosigkeit und Perspektivlosigkeit, die insbesondere junge Männer in Identitätskrisen stürzen. Sie können die Rollenerwartungen nicht erfüllen und suchen folglich nach Kompensationen; dann dient ihnen die Teilhabe an der Kriegsökonomie als willkommene Möglichkeit zur Existenzsicherung. Neue militärische Rituale, die Rekruten in Armeen oder Guerillagruppen zusammenschweißen sollen, werden als identitätsstabilisierend vermittelt; wenn der Einzelne diese durchsteht, hat er seine Mannhaftigkeit bewiesen. Allerdings sind die oft sadistischen und sexistischen Initiationsrituale keineswegs auf die Herstellung von Egalität, sondern auf Hierarchien ausgerichtet. Rekruten, die sich den ritualisierten Demütigungen durch Ranghöhere verweigern, werden als verweiblichte Feiglinge verhöhnt. $\mathrm{Zu}$ dem setzen ranghohe Militärs und Kriegsherren männliche Ehre mit Kampfbereitschaft gleich und lenken Männerfreundschaften in militärische Kameraderie um; gleichzeitig etablieren sie durch militärische Ränge neue Hierarchien zwischen Männern (Schäfer 2008:7ff.). Diese grundlegenden Muster forcierten die Militarisierung von Männlichkeit und die hohe Gewaltbereitschaft in Sierra Leone und Uganda.

\section{Gender im Bürgerkrieg in Sierra Leone}

Der Bürgerkrieg im westafrikanischen Küstenstaat Sierra Leone begann 1991 und dauerte bis Ende 2001. Dort kämpften Regierungstruppen gegen eine Guerillabewegung, die sogenannte Revolutionary United Front (RUF). Deren Anführer und Hintermänner, allen voran der frühere Warlord und liberianische Präsident Charles Taylor, waren vor allem an der eigenen Bereicherung interessiert. Sie rekrutierten Jugendliche mit vagen Versprechungen vom Zugang zu Bildung und dem Aufbau einer gerechten Gesellschaft. Dabei instrumentalisierten die Drahtzieher der RUF die in der Geschichte des Landes begründeten politischen Strukturprobleme und die kolonial geprägten Ausbeutungsverhältnisse für ihre Propaganda. Bereits ab 1787 hatten die britischen Kolonialherren Nachfahren freigelassener Sklaven aus Amerika, sogenannte Kreolen, an der Küste angesiedelt. Deren Bevorzugung in Bildung, Wirtschaft und Politik sorgte immer wieder für Konflikte; diese wurden durch die Einsetzung neuer lokaler Autoritäten im Landesinneren durch die Kolonialbeamten verstärkt. Dort waren junge Männer in jeder Hinsicht von der Gunst der alten und einflussreichen Familienoberhäupter abhängig; das betraf vor allem den Zugang zu Land und die Eheschließungen. Oft mussten sich die Jungen jahrelang auf den Feldern wohlhabender Landbesitzer verdingen und alte Männer heirateten junge Mädchen, mit denen junge Männer bereits geheime Liebesbeziehungen aufgebaut hatten (Schäfer 2008:217ff.).

Von den ab den 1920er Jahren in den südlichen Landesteilen angebauten Exportprodukten wie Kaffee oder Kakao profitierte nur eine kleine lokale Elite. Auch nach der politischen Unabhängigkeit 1961 investierten die neuen politischen Machthaber die Gewinne aus dem lukrativen Diamantenabbau nicht zum Wohl der Bevölkerung. Anstatt Entwicklungsprogramme gegen die fortschreitende Verarmung aufzulegen, veruntreuten sie die Devisen in großem Stil. Wiederholt schlugen Polizei und Armee Proteste gewaltsam nieder, zudem verspielten Polizisten und Richter durch Willkür, Inkompetenz und Bestechlichkeit das Vertrauen der Bevölkerung. Einbrüche der Weltmarktpreise für Kaffee und Kakao sowie die vom Internationalen Weltwährungsfonds verordneten wirtschaftlichen Strukturanpassungsprogramme trieben das Land immer weiter in die Krise. Ende der 1980er Jahren wurde der geringe Gesundheits- und Bildungsetat abermals drastisch gekürzt. Immer mehr jungen Menschen blieben jegliche Zukunftsperspektiven verwehrt. Diese Exklusionserfahrungen boten die Grundlage für die Rekrutierung von Jugendlichen durch RUF-Milizen ab Anfang der 1990er Jahre (Richards 2006:183ff.). Sie griffen keineswegs nur wegen korrupter Politiker und Staatsdiener, nicht-funktionierender staatlicher Institutionen und der maroden Wirtschaft zu den Waffen. Sie wollten auch die Macht der alten Männer auf lokaler Ebene brechen.

Dennoch kamen viele Jugendliche nicht freiwillig zur RUF; mehrheitlich wurden sie zwangsrekrutiert. Dabei setzten die RUF-Kommandanten systematisch sexualisierte Demütigungsrituale ein. Diese traumatische Gewaltsozialisation, verstärkt durch die Verabreichung harter Drogen, führte dazu, dass RUFKämpfer besonders grausam gegen diejenigen vorgingen, die ihre Kommandanten oft willkürlich als „Feinde“ titulierten. Mancherorts betraf dies die gesamte Dorfbevölkerung. Eine systematisch eingesetzte Kampftaktik waren brutale (Massen) Vergewaltigungen; dadurch wurden keineswegs nur die Opfer schwer verletzt, sondern auch die „feindlichen“ Männer als Versager verhöhnt. Darüber hinaus ordneten jugendliche RUFKommandanten Vergewaltigungen an, um den Zusammenhalt ihrer Untergebenen zu festigen. Innerhalb der RUF-Hierarchien eigneten sie sich Privilegien an, über die zuvor nur alte und reiche Männer verfügten, insbesondere die Kontrolle über Frauen und Mädchen. Schätzungen gingen davon aus, dass die RUFGuerillaeinheiten während des Krieges über 70.000 Jugendliche rekrutierten. Der Anteil der Frauen und Mädchen betrug zwischen zehn und dreißig Prozent. Die meisten Rekrutinnen mussten Spionage-, Boten-, Träger-, Koch- und Pflegedienste leisten; teilweise wurden sie als Kämpferinnen eingesetzt. Die RUF-Kommandanten bauten eine neue Rangordnung zwischen den jungen Mädchen auf, indem sie einige wenige als sogenannte „bush wives“ - also als temporäre Partnerinnen auswählten, und die Mehrheit zu Sex-Sklavinnen degradierten, die allen Kämpfern zur Verfügung stehen mussten.

Eigentlich hatte die RUF gegenüber der schwachen und miserabel ausgerüsteten sierra-leonischen Armee ein leichtes Spiel. Während der Kriegsjahre gab es wiederholt Putsche und etliche 
Soldaten wechselten mehrfach die Fronten. Die internationale Staatengemeinschaft unternahm jahrelang keine Anstrengungen zur Konfliktbeilegung, deshalb wandte sich der damalige General Valentine Strasser an den privaten südafrikanischen Sicherheitsdienst Executive Outcomes (EO). Dieser schwächte die RUF so weit, dass sie schließlich in Friedensverhandlungen einwilligte. Allerdings setzten die Rebellen bereits kurz nach der Unterzeichnung des Friedensvertrags von Abidjan Ende November 1996 ihre Kämpfe fort. Nach langem Ringen unterzeichneten die Kriegsakteure am 7. Juli 1999 das Friedensabkommen von Lomé. In der neugebildeten Regierung übernahmen RUF-Vertreter ranghohe Posten, die ihnen die Kontrolle über die Rohstoffe des Landes sicherten. Durch den lukrativen Diamantenschmuggel verschaffte sich die RUF weiterhin zusätzliche Einnahmen, wobei nicht nur Milizen im Libanon, sondern offenbar auch Mitglieder des Terrornetzwerks El Kaida in den illegalen Diamantenhandel involviert waren (Schäfer 2008:243).

\section{UN-Interventionen und Nachkriegsprobleme in Sierra Leone}

Am 22. Oktober 1999 erließ der UN-Sicherheitsrat die Resolution 1270. Sie bot die Grundlage für die UN-Mission in Sierra Leone (UNAMSIL) mit 6.000 Blauhelmsoldaten. Allerdings waren etliche UN-Einheiten technisch unzureichend ausgestattet und verfügten kaum über Landeskenntnisse. Diesen Umstand nutzte die weiterhin aktive RUF aus, indem sie kurz darauf über 500 sambische Blauhelmsoldaten gefangen nahm. Im Mai 2000 empfahl der damalige UN-Generalsekretär Kofi Annan, die Friedenstruppe auf 16.500 Blauhelmsoldaten zu erhöhen; drei Monate später legte er eine erneute Aufstockung um weitere 1.000 Mann nahe, dann waren 17.500 Soldaten im Einsatz. Angesichts der gefährlichen und verworrenen Konfliktsituation kompensierten etliche der internationalen Einsatzkräfte ihre Verunsicherung durch sexuelle Kontakte mit sierra-leonischen Mädchen. Während ihr Militäreinsatz lange Zeit von geringem Erfolg gekrönt war, konnten sie zumindest gegenüber der lokalen männlichen Bevölkerung mit ihrem besitzergreifenden Sexualverhalten auftrumpfen. Zahlreiche Kommandanten der Friedenstruppen duldeten dieses Vorgehen und verhielten sich sogar selbst wie Sextouristen. Ihr Macht- und Potenzgebaren war insbesondere für junge Männer ein Affront, allerdings straften sie nicht die Blauhelmsoldaten selbst, sondern die beteiligten Mädchen ab (Higate / Henry 2004:496ff.).

Mangels Einkommensalternativen sahen sich vor allem sozial marginalisierte frühere RUF-Kämpferinnen zu sexuellen Kontakten mit den Blauhelmsoldaten gezwungen. Im Rahmen der Demobilisierung hatten RUF-Kommandanten ihnen die Waffen abgenommen; sie wollten nicht als Anführer von Frauen und Kindern gelten. Obwohl die RUF mehrere zehntausend Kombattantinnen rekrutiert hatte, beschränkte sich die Zahl der Demobilisierten auf etwa 4.000 bis 4.700 Frauen und 500 bis 1.600 Mädchen. Ex-Kommandanten wählten bestenfalls eine ihrer „bush wives “ für die Registrierung im DDR-Programm aus. Alle anderen „bush wives“ und Sex-Sklavinnen blieben von den DDR-Förderprogrammen ausgeschlossen. Sie hatten im
Nachkriegskontext besonders große Probleme, ihre Existenz zu sichern. Daher versuchten einige, Landnutzungsrechte durch eine Eheschließung zu erhalten; meist verheimlichten sie ihre Kriegserlebnisse gegenüber ihren Partnern. Viele Ehemänner reagierten mit Gewalt, wenn sie durch Verhaltensauffälligkeiten oder Krankheiten ihrer Ehefrauen dennoch damit konfrontiert wurden. Darüber hinaus verlangten alte einflussreiche Frauen, über sexuelle Kriegsgewalt zu schweigen. Sie zwangen Ex-Kämpferinnen zu genitalen Beschneidungen, wobei sie diese Verstümmelungen als Inbegriff von Kultur und Tradition ausgaben. Gemeinsam mit alten ranghohen Männern pochten sie darauf, dass nur durch die Rückkehr zu Traditionen der Wiederaufbau der sozialen Ordnung möglich sei. Solche Einschätzungen bestärkten Männer, die im Krieg den Vergewaltigungen ihrer Ehefrauen hilflos zuschauen mussten, fortan ebenfalls gewaltsam ihre eheliche Vormachtstellung einzufordern oder sogar ihre Frauen wegzuschicken.

Trotz des geforderten Schweigens über Vergewaltigungen wird die Zahl der Gewaltopfer während des Krieges in Sierra Leone landesweit auf 215.000 bis 270.000 Frauen und Mädchen geschätzt; Massenvergewaltigungen überlebten etwa ein Drittel der Frauen. Mindestens 90.000 wurden mit HIV infiziert - und das bei einer Gesamtbevölkerung von insgesamt etwa fünf Millionen. Über 20.000 Kinder waren durch Vergewaltigungen gezeugt worden (Human Rights Watch 2003a:25ff.). Nur wenige städtische Nicht-Regierungsorganisationen boten den Teenager-Müttern Hilfe an. Vor allem Ex-RUF-Kombattantinnen sahen sich zur Prostitution gezwungen, um so heftiger wurden sie von ihrem sozialen Umfeld angefeindet.

Im Rahmen der Demobilisierung und Reintegration blieben gewaltgeprägte Männlichkeitsvorstellungen weitgehend unangetastet. Zahllose demobilisierte Ex-Kombattanten sind bis heute in ihrem Selbstbild verunsichert und nutzen auch weiterhin Gewalt, um ihre Autorität zu festigen. Die Hoffnungen junger unverheirateter Männer auf eine bessere Zukunft wurden mehrheitlich enttäuscht. Selbst diejenigen, die Landwirtschaftskurse zur Reintegration absolvierten, erhielten oft keinen Landzugang. Vertreter der alten Elite, die bereits zu Kriegsbeginn geflohen waren, eigneten sich Entwicklungsgelder an, weil zahllose Hilfsorganisationen in Unkenntnis der lokalen Hierarchien mit ihnen kooperierten und damit alte Machtmuster reproduzierten.

Auch beim Wiederaufbau staatlicher Institutionen wie Polizei und Justiz sind Nepotismus und Korruption bereits wieder Strukturprobleme. Daran konnten die übereilt eingesetzte Wahrheits- und Versöhnungskommission und das Sondertribunal nichts ändern. Beide trugen nur ansatzweise zur erhofften Aufdeckung der Gräueltaten und zur Bestrafung von Kriegsverbrechern bei. Obwohl es einige Rechtsreformen gab und häusliche Gewalt ebenso wie Vergewaltigungen in Sierra Leone inzwischen Straftatbestände sind, werden nur wenige Gewaltakte strafrechtlich verfolgt. Vielerorts gibt es keine Ausbildungsmöglichkeiten, da Vertreter der lokalen Verwaltung und des Schulsystems nach wie vor inkompetent sind und systematisch Gelder veruntreuen. Mangels Alternativen sind viele junge Männer gezwungen, sich als schlecht bezahlte Diamantenschürfer zu verdingen, als Kleinkriminelle in den Städten ihre Existenz zu sichern oder als Söldner in einem Nachbarland 
weiterzukämpfen. Die dazu notwendigen Kleinwaffen sind reichlich vorhanden (Quee 2008:3ff.).

\section{Gender und Bürgerkrieg in Nord-Uganda}

Auch im Norden Ugandas sorgen vielschichtige Generationen- und Geschlechterkonflikte für gesellschaftliche Strukturprobleme. Diese reichen weit in die Kolonialzeit zurück. Hier kooptierten die britischen Kolonialherren ab Ende des 19. Jahrhunderts Vertreter des Buganda-Königreiches im fruchtbaren und regenreichen Süden des Landes für ihre Verwaltung. Gleichzeitig rekrutierten sie junge Männer aus den Nomadenvölkern im Norden als Kolonialsoldaten und projizierten eine besonders martialische Männlichkeit auf diese Nomadengruppen. Landwirtschaftliche Förderprogramme, die dem Süden zu weiterer Blüte verhalfen, blieben den Bewohnern nördlicher Landesteile vorenthalten. Nach der politischen Unabhängigkeit Ugandas 1962 erhielten diese Gegensätze eine zusätzliche Dynamik, zumal Milton Obote und Idi Amin sich als despotische Machthaber etablierten und ihre Soldaten aus verschiedenen Völkern im Norden des Landes rekrutierten.

Anfang der 1980er Jahre begann Yoweri Museveni einen Guerillakrieg gegen die Diktatur Obotes; im Januar 1986 übernahm er die Macht. Obotes Truppen, allen voran Acholi-Soldaten, wurden rasch demobilisiert und in eine unsichere Zukunft entlassen. Von den geplanten Entwicklungsprogrammen im Norden wurde nur ein Bruchteil realisiert. So blieben viele demobilisierte Soldaten arbeitslos und hatten Probleme, sich wieder in ihre Herkunftsgesellschaft einzufügen. Sie waren durch den jahrelangen Militärdienst und den brutalen Krieg gegen Museveni verroht. Wegen zahlreicher Gewaltübergriffe auf ihre eigenen Familienangehörigen und ihrer Respektlosigkeit gegenüber Älteren wurden ihnen traditionelle Reinigungsrituale, mit denen Mörder in die dörfliche Gemeinschaft reintegriert werden konnten, verweigert. Bereits 1986 bot das Geistermedium Alice Auma den demobilisierten Acholi-Soldaten neu erfundene Heilrituale an und mobilisierte sie zum erneuten Kampf gegen Museveni. Dazu fühlte sich Alice Auma nach eigenen Angaben von einem christlichen Geist berufen, weshalb sie ihre Guerillagruppe Holy Spirit Mobile Forces (HSMF) nannte. Die religiösen Heilsversprechungen veranlassten einige Männer, sich den HSMF anzuschließen, obwohl diese Gruppe von einer Frau geleitet wurde. Beim Vormarsch auf Kampala 1987 rekrutierte die Gruppe viele Mitstreiter gewaltsam, so dass ihre Zahl auf 7.000 bis 10.000 anwuchs. Allerdings wurde die HSMF bereits 50 Kilometer vor der Hauptstadt von der ugandischen Armee geschlagen. Über den Verbleib Alice Aumas gibt es nur Spekulationen; manche Beobachter berichten, sie sei nach Kenia geflohen (Allen 2006:36).

Kurz darauf tauchte Josef Kony als neuer Anführer einer weiteren religiös legitimierten, militanten Gruppe in Nord-Uganda auf. Er gründete die Lord's Resistance Army (LRA), für die er neben demobilisierten Acholi-Soldaten über 20.000 Kinder zwangsrekrutierte, darunter 7.500 Mädchen. Sexualisierte Gewalt zählte zu seinen Rekrutierungsstrategien, wobei Mädchen und Jungen sowohl zu Opfern als auch zu Tätern wurden. Nur wenige Mädchen wurden militärisch trainiert und zu Fronteinsätzen geschickt. Auch wenn sie gelegentlich an Überfällen teilnehmen mussten, verlangten die LRA-Kommandanten von ihnen vor allem Dienste als Trägerinnen, Köchinnen oder SexSklavinnen. Kony und seine Kommandanten ordneten sexuelle Gewalt und Massenvergewaltigungen an. Auch sie bauten eine Hierarchie zwischen „bush wives“ und Sex-Sklavinnen auf. Über 1.000 dieser Kindersoldatinnen wurden schwanger und brachten selbst Kinder zur Welt (Schäfer 2008:348f.).

Kony unterstellte seinen Gegnern, mit bösen Geistern zu paktieren und verlangte von seinen Untergebenen, alle Feinde zu verstümmeln oder zu ermorden. Ab 1990 belieferte die sudanesische Regierung die LRA mit Waffen, um gegen Museveni vorzugehen. Sie warfen ihm vor, die südsudanesische Befreiungsbewegung zu unterstützen. Mehrere Phasen unterschiedlicher Kampfintensität kennzeichneten die Auseinandersetzungen zwischen LRA und ugandischen Regierungstruppen zwischen Juni 1988 und März 1994. In dem Zeitraum fanden jedoch auch wiederholt Friedensverhandlungen statt.

Etliche Regierungssoldaten traten nicht als Beschützer auf, sondern schürten durch eigene Gewaltakte das Misstrauen in der Zivilbevölkerung. 1999 unterzeichneten die ugandische und die sudanesische Regierung ein Friedensabkommen, bei dem sich beide Seiten dazu verpflichteten, jegliche Unterstützung für die Guerillas in der gesamten Region einzustellen. Jedoch versorgten einflussreiche sudanesische Kommandanten Kony weiterhin mit Waffen. Auch einzelne ranghohe ugandische Militärs hatten kein Interesse an einer Konfliktbeilegung, denn jahrelang strichen sie mit fingierten Soldatenzahlen hohe Soldzahlungen persönlich ein. Viele Soldaten vermieden ein hartes Durchgreifen, da sie den Machtgewinn der LRA als Angelegenheit der Bewohner Nordugandas betrachten und dafür nicht ihr Leben riskieren wollten. Im Jahr 2000 erließ Museveni eine Generalamnestie für die LRA und integrierte übergelaufene Kämpfer in seine Armee. Im März 2002 sollten die Regierungstruppen zu einem neuen Militärschlag ausholen, doch auch der blieb erfolglos. Ende 2003 beantragte der ugandische Staatspräsident beim Internationalen Strafgerichtshof (ICC) Ermittlungen gegen Kony und andere LRA-Anführer. Anfang Oktober 2005 stellte der Chefankläger des ICC mehrere Haftbefehle aus. Zwischenzeitlich wurden wiederholt Waffenstillstands- und Friedensabkommen ausgehandelt, die Kony aber nicht einhielt (Maina 2009:49f.). Vertreter von Kirchen, Frauen-, und Menschenrechtsgruppen stritten darüber, inwieweit die strafrechtliche Verfolgung ein Ende der Gewalt bringen könnte, zumal die Täter nicht gefasst wurden. Die unterschiedlichen zivilgesellschaftlichen Gruppen hatten konträre Vorstellungen von Strafe, Sühne und Gerechtigkeit: Manche plädierten dafür, gerichtliche Strafen mit kulturell angepassten Versöhnungsritualen zu verbinden. Andere meinten, die LRA sei ohne militärischen Druck nicht zu Friedensgesprächen bereit. Inzwischen operiert Kony von der Zentralafrikanischen Republik bzw. der Demokratischen Republik Kongo aus, wo seine Kämpfer ebenfalls Zivilisten massakrieren. 


\section{Gender in ugandischen Flüchtlingslagern}

Ab April 1994 richtete die ugandische Regierung in großem Stil sogenannte „protected villages“ für die Bewohner abgelegener Siedlungen in den nördlichen Landesteilen ein. Hier sollten sie vor Übergriffen durch die LRA und bei Kampfhandlungen zwischen LRA und Regierungsarmee geschützt werden. Gleichzeitig dienten die von Regierungssoldaten bewachten Lager dazu, die Versorgung der LRA durch Überfälle auf Gehöfte zu unterbinden. Allerdings boten die sehr beengten und nur rudimentär ausgestatteten „Schutzdörfer“ den Flüchtlingen wenig Sicherheit. 1998 waren ca. 400.000 Menschen in Nord-Uganda auf der Flucht oder wurden umgesiedelt. 2004 stieg die Zahl auf 800.000; 2006 lebten in den „Schutzdörfern“ 1,2 Millionen Menschen; faktisch betraf das die Mehrheit der Acholi-Bevölkerung (Allen 2006:53f.).

Angesichts der mangelhaften Versorgung sahen sich etliche Frauen für den Zugang zu Lebensmitteln zu Sex mit Lagerleitern und Regierungssoldaten gezwungen, was die Verbreitung von HIV/AIDS und Geschlechtskrankheiten zur Folge hatte. Viele Ehemänner und Väter, die ihr Vieh und eigene Einkommensmöglichkeiten verloren hatten, gerieten in schwere Identitätskrisen. Sie konnten ihre Familie nicht versorgen und beschützen, weshalb sie von etlichen Regierungssoldaten herablassend behandelt wurden. Auf diese Verachtung reagierten zahlreiche Familienväter wiederum mit häuslicher Gewalt, um ihrer ehelichen Autorität Ausdruck zu verleihen (Human Rights Watch 2003b:36ff.).

Junge, unverheiratete Männer fühlten sich durch das Potenzgebaren der vergleichsweise zahlungskräftigen Regierungssoldaten in ihrer Männlichkeit gedemütigt. Manche waren zuvor von der LRA rekrutiert worden und konnten fliehen; so kamen sie in die Flüchtlingslager. Nun fehlten ihnen die wirtschaftlichen Grundlagen, um die hohen Brautpreisforderungen der älteren Familienväter aufzubringen. Brautgaben waren aber notwendig, um eine legale Ehe schließen zu können und als vollwertiger Mann respektiert zu werden. Andernfalls wurden sie - selbst wenn sie bereits Kinder gezeugt hatten - im Machtgefüge zwischen Männern weiterhin als unmündig angesehen (Dolan 2002:59ff.). Nicht wenige dieser Männer „bestraften“ junge Mädchen, die Beziehungen mit Soldaten eingingen, mit sexueller Gewalt. So tyrannisierten etwa Brüder ihre eigenen Schwestern, um auf diese Weise Macht zu erleben; andere schlossen sich sogar selbst der Armee an.

Auch die Gewalt unter Männern eskalierte, wobei lokale Generationenkonflikte mit politischen Konflikten verwoben waren. Etliche Acholi-Älteste hatten nach der Machtübernahme Musevenis 1986 junge Männer zum Kampf aufgerufen. Als die so motivierten Gewaltakte durch Freiwillige in der HSMF und der LRA außer Kontrolle gerieten, bestritten sie, jemals die Jugendlichen zur Gewalt ermutigt zu haben (Finnström 2006:210). Inzwischen fühlen sich die jungen Männer von den sogenannten „Veranda-Ältesten“ verraten, die sie sogar als Unruhestifter anfeinden. Viele Förderprogramme der Regierung und internationaler Geber bleiben den Jugendlichen vorenthalten, so dass die zornigen und gewaltbereiten jungen Männer aus der Sicht kritischer Beobachter auch hier weiterhin ein latentes und strukturelles Sicherheitsproblem darstellen.

\section{Fazit}

Die hier erläuterten Zusammenhänge verdeutlichen, wie wichtig es ist, Gender als strukturprägendes Machtphänomen vor, während und nach Kriegen zu erkennen und die vielschichtigen Differenzen zwischen Männern und zwischen Frauen in Forschungen über Sicherheitsfragen zu ergründen. So sind feministische Positionen zu relativieren, zumal nicht alle Frauen in gleicher Weise Gewaltopfer werden und Konflikte zwischen Frauen unterschiedlichen Alters und Status idealisierende Vorstellungen von einer verbindenden Solidarität ins Wanken bringen. Die wissenschaftliche Auseinandersetzung mit den Unterschieden zwischen Frauen im konkreten empirischen Kontext wäre eine Voraussetzung dafür, angepasste Programme für die jeweiligen Gewaltopfer zu konzipieren.

Besonders deutlich wird die Relevanz von Gender als Analysekategorie für Gewaltprobleme, wenn man die Unterschiede und Hierarchien zwischen Männern näher beleuchtet. Dabei ist es wichtig, die Distinktionen zwischen Männern unterschiedlicher Herkunft zu erfassen, beispielsweise zwischen Blauhelmsoldaten und lokaler männlicher Bevölkerung. Zudem sollten Macht-, Interessen- und Generationskonflikte zwischen Männern, z.B. zwischen Soldaten der Regierungsarmee und jungen, verarmten Männern, untersucht werden, denn sie beeinflussen deren Gewalthandeln und die Fortsetzung militarisierter Männlichkeit in Nachkriegsgesellschaften. Diese Probleme sind in Flüchtlingslagern besonders virulent.

Vor allem demobilisierte, demoralisierte und perspektivelose Ex-Kämpfer fordern ihre eheliche Dominanz ein. Ehefrauen, die während eines Krieges von feindlichen Truppen vergewaltigt wurden, werden im Nachhinein von ihren Ehepartnern gedemütigt oder fortgeschickt. Die Frauen gelten auch als gefährliches Krankheitsrisiko, weil sie möglicherweise mit Geschlechtskrankheiten oder HIV infiziert wurden, und als Bedrohung der familiären Erbfolge, wenn sie Kinder zur Welt bringen, deren Väter man nicht kennt. Gelegentlich bedrängen ältere Frauen ihre Söhne, sich von den vergewaltigten Ehefrauen zu trennen.

Darüber hinaus sind vor allem Ex-Kämpferinnen mit Gewalt konfrontiert. Vielerorts halten Kommandanten sie davon ab, an Demobilisierungsprogrammen teilzunehmen. Wegen ihrer ambivalenten Rolle - als Frauen, die zwar selbst Gewalt erlitten, aber auch getötet haben - können Ex-Kombattantinnen nicht mit Unterstützung durch andere Frauen in Nachkriegsgesellschaften rechnen. Häufig konkurrieren sie in der umkämpften Nachkriegsökonomie mit ihren Geschlechtsgenossinnen und werden von ihnen als Prostituierte angefeindet.

Wenn sexualisierte Kriegsgewalt nicht strafrechtlich verfolgt, von politischen Entscheidungsträgern geächtet und gesellschaftlich aufgearbeitet wird, besteht die Gefahr, dass sie ungebrochen in Nachkriegsgesellschaften übernommen wird. In Zeiten umfassender Entwaffnung, die viele demobilisierte Männer als Kontrollverlust erleben, ist sexualisierte Gewalt ein Machtmittel zur maskulinen Selbstbestätigung. Das hat, neben den direkten physischen und psychischen Verletzungen, die den Opfern zugefügt werden, auch einen weiteren Anstieg von HIV-Infektionen und Geschlechtskrankheiten zur Folge. Um 
so problematischer ist das besitzergreifende Sexualverhalten von Blauhelmsoldaten, die durch ihr Potenzgebaren die lokale männliche Bevölkerung, vor allem Ex-Kombattanten, provozieren. Zwar hat die UN für ihre Blauhelmsoldaten strenge Verhaltensrichtlinien erlassen, Verstöße werden jedoch nur selten geahndet.

Innovative Strategien zur Überwindung kriegsgeprägter Männlichkeit erfordern es, die Motive zur (un)freiwilligen Kriegsbeteiligung junger Männer, ihre komplexen Kriegserfahrungen als Täter und Opfer sowie die Auswirkungen auf sie aufzuarbeiten. Notwendig sind auch kritische Auseinandersetzungen mit extremen Ungleichheiten im Zugang zu Land und anderen Ressourcen, mit Machtmissbrauch durch politische Autoritäten, mit Korruption und Behördenwillkür. Land-, Rechts- und Verwaltungsreformen sollten jungen Männern Chancen zur wirtschaftlichen Eigenständigkeit und politischen Partizipation geben (Akude 2009:243). Allerdings sind institutionelle Strukturveränderungen nur ein Schritt, um die kriegerische Gewaltprägung junger Männer zu überwinden. Gleichzeitig sind politische und religiöse Autoritäten auf nationaler und lokaler Ebene gefordert, junge Männer nicht für ihre eigenen Machtinteressen zu instrumentalisieren und nach Kriegen heuchlerisch deren Gewaltbereitschaft anzuprangern.

\section{Literatur}

Akude, John Emeka 2009, Unfrieden nach Rezept, in: Entwicklung und Zusammenarbeit, 50:6, 240-243.

Allen, Tim 2006, Trial justice, The International Criminal Court and the Lord's Resistance Army, London.

Dolan, Chris 2002, Collapsing masculinities and weak states, A case study of Northern Uganda, in: Cleaver, Frances (ed.): Masculinities matter! Men, gender and development, London, 57-83.
Finnström, Sverker 2006, Meaningful rebels? Young adult perception on the Lord's Resistance Movement/Army in Uganda, in: Christiansen, Catrine / Utas, Mats / Vigh, Henrik (eds.): Navigating youth, generating adulthood, Social becoming in an African context, Uppsala, 203-227.

Higate, Paul / Henry, Marsha 2004, Engendering (in)security in peace support operations, in: Security Dialogue, 35:4, 481498.

Human Rights Watch 2003a, "We'll kill you if you cry", Sexual violence in the Sierra Leone conflict, Human Rights Watch Publications, vol. 15, no. 1 (A), New York.

Human Rights Watch 2003b, "Just die quietly", Domestic violence and women's vulnerability to HIV in Uganda, Human Rights Watch, vol. 15, no. 15, New York.

Maina, Grace 2009, Questioning reintegration processes in Northern Uganda, in: Conflict Trends, 1, 48-54.

Richards, Paul 2006, Forced labour and civil war, Agrarian underpinnings of the Sierra Leone conflict, in: Kaarsholm, Preben (ed.): Violence, political culture and development in Africa, Oxford, 181-200.

Quee, Allan 2008, Regional child warriors in Africa, in: Conflict Trends, 3, 3-10.

Schäfer, Rita 2008, Frauen und Kriege in Afrika, Ein Beitrag zur Gender-Forschung, Frankfurt a.M.

Ulbert, Cornelia 2008, Menschliche Sicherheit = männliche Sicherheit? Der Einfluss von Gender und Identität(en) auf die kulturelle Konstruktion von (Un-)Sicherheit, in: Ulbert, Cornelia / Werthes, Sascha (Hrsg.): Menschliche Sicherheit - Globale Herausforderungen und regionale Perspektiven, Baden-Baden, 51-65.

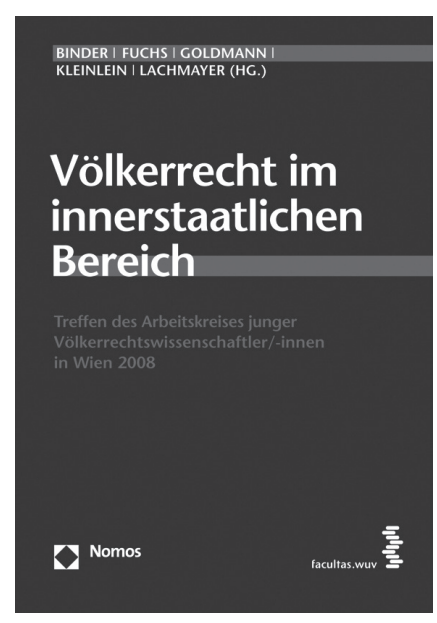

\section{Völkerrecht im innerstaatlichen Bereich}

Treffen des Arbeitskreises junger Völkerrechtswissenschaftler/-innen in Wien 2008 Herausgegeben von Christina Binder, Claudia Fuchs, Matthias Goldmann,

Thomas Kleinlein und Konrad Lachmayer

2010, 216 S., brosch., 36,- €, ISBN 978-3-8329-5218-1

(Schriften zum Internationalen und Vergleichenden Öfentlichen Recht, Bd. 13)

Der Tagungsband behandelt die Wechselbeziehungen zwischen staatlichem Recht und der Völkerrechtsordnung. 